\title{
Incidence of Malignancies in Patients with IgG4-related Disease
}

\author{
Kenji Hirano ${ }^{1}$, Minoru Tada ${ }^{1}$, Naoki Sasahira ${ }^{1}$, Hiroyuki Isayama ${ }^{1}$, Suguru Mizuno ${ }^{1}$, \\ Kaoru Takagi ${ }^{1}$, Takeo Watanabe ${ }^{1}$, Tomotaka Saito ${ }^{1}$, Shuhei Kawahata ${ }^{1}$, Rie Uchino ${ }^{1}$, \\ Tsuyoshi Hamada ${ }^{1}$, Koji Miyabayashi ${ }^{1}$, Dai Mohri ${ }^{1}$, Takashi Sasaki ${ }^{1}$, Hirofumi Kogure ${ }^{1}$, \\ Natsuyo Yamamoto ${ }^{1}$, Yousuke Nakai ${ }^{1}$, Haruhiko Yoshida ${ }^{1}$, Yukiko Ito $^{2}$, Dai Akiyama ${ }^{2}$, \\ Nobuo Toda ${ }^{3}$, Toshihiko Arizumi ${ }^{3}$, Hiroshi Yagioka ${ }^{4}$, Naminatsu Takahara ${ }^{5}$, \\ Saburo Matsubara ${ }^{6}$, Yoko Yashima $^{7}$ and Kazuhiko Koike ${ }^{1}$
}

\begin{abstract}
Objective It has been discussed whether IgG4-related disease (IgG4-RD), including autoimmune pancreatitis (AIP), is associated with malignancy; however, the issue has not been clarified.

Methods We analyzed 113 patients with IgG4-RD in whom malignancy was not diagnosed at the time of IgG4-RD onset and the follow-up period was longer than six months. A total of 95 patients had AIP. The mean follow-up period was 73 months. The incidence of the observed malignancies was compared with the expected incidence in an age- and sex-matched general Japanese population based on the Vital Statistics of Japan.

Results There were 15 malignancies (lung cancer in five patients, pancreatic cancer in two patients, gastric cancer in two patients, bile duct cancer in one patient, renal cancer in one patient, breast cancer in one patient, tongue cancer in one patient, malignant melanoma in one patient and acute myeloid leukemia in one patient) in 14 patients during the follow-up period. The calculated standardized incidence rate of the total malignancies was not significant, that is, 1.04 (95\% CI 0.57-1.75).

Conclusion The incidence of total malignancies in IgG4-RD patients is similar to that observed in the general population. At present, it is reasonable to conclude that IgG4-RD is not associated with an increased incidence of total malignancies.
\end{abstract}

Key words: IgG4-related disease, autoimmune pancreatitis, malignancy, diabetes mellitus

(Intern Med 53: 171-176, 2014)

(DOI: 10.2169/internalmedicine.53.1342)

\section{Introduction}

Several case reports of malignancy in patients with autoimmune pancreatitis (AIP) and IgG4-related disease (IgG4RD) have been published (1). For example, we previously reported the occurrence of malignancy in three of 42 patients with AIP (pancreatic cancer in one patient, lung can- cer in one patient and acute myeloid leukemia in one patient) (2). Kubota et al. described two cases of pancreatic cancer and two additional cases of other cancer (one case of breast cancer and one case of rectal cancer) (3). Takuma et al. reported the occurrence of cancer in six of 50 patients with AIP (lung cancer in four patients, esophageal cancer in one patient and prostate cancer in one patient) (4). Therefore, in addition to pancreatic cancer, several other malig-

\footnotetext{
${ }^{1}$ Department of Gastroenterology, Graduate School of Medicine, University of Tokyo, Japan, ${ }^{2}$ Department of Gastroenterology, Japanese Red Cross Medical Center, Japan, ${ }^{3}$ Department of Gastroenterology, Mitsui Memorial Hospital, Japan, ${ }^{4}$ Department of Gastroenterology, JR Tokyo General Hospital, Japan, ${ }^{5}$ Department of Gastroenterology, Kanto Central Hospital of the Mutual Aid Association of Public School Teachers, Japan, ${ }^{6}$ Department of Gastroenterology, Tokyo Metropolitan Police Hospital, Japan and ${ }^{7}$ Department of Hepatology, Kyoundo Hospital, Japan Received for publication July 10, 2013; Accepted for publication August 20, 2013 Correspondence to Dr. Kenji Hirano, khirano-tky@umin.ac.jp
} 
nancies have been observed in patients with AIP. It is not unexpected that the processes of chronic inflammation and fibrosis observed in patients with IgG4-RD, including AIP, may increase the risk of malignancy. However, whether these malignancies are merely attributable to an older age remains unknown. In this study, we aimed to evaluate the incidence of malignancy in patients with IgG4-RD during follow-up, compared with that observed in the general Japanese population. We also aimed to clarify which factors are correlated with the occurrence of malignancy in IgG4-RD patients.

\section{Materials and Methods}

Since Yoshida et al. reported the concept of AIP in 1995 (5), various diagnostic criteria for AIP have been proposed (6-10). In this study, patients with type 1 AIP (IgG4related AIP) whose diagnosis was eventually confirmed based on the international consensus diagnostic criteria (ICDC) (10) were enrolled. Among patients without AIP, those with IgG4-RD whose diagnosis was confirmed based on comprehensive diagnostic criteria for IgG4-RD (11) were enrolled. Patients in whom malignancy was diagnosed $\leq 6$ months before or after the onset of IgG4-RD were excluded. Patients whose follow-up period was $\leq 6$ months were also excluded.

In total, 126 patients were diagnosed with IgG4-RD at our institute and affiliated hospitals between January 1997 and September 2012. In seven patients, malignancy was diagnosed $\leq 6$ months before or after the onset of IgG4-RD. The follow-up period was $\leq 6$ months in six patients. After excluding these 13 patients, 113 patients were enrolled in this study. These patients were followed carefully every 3-6 months, and clinical data were prospectively collected. Chest and abdominal contrast-enhanced computed tomography (CT) was performed once or twice each year. Plain CT was performed when contrast medium could not be used. When patients showed any signs suggestive of malignancy, such as weight loss, anemia or tumor marker elevation, they underwent additional examinations, such as colonoscopy, gastroduodenoscopy, chest X-ray, CT and positron emission tomography, according to the suspected malignancy. In patients without any signs or symptoms of malignancy, we recommended the use of a screening test once a year during the follow-up, particularly for cancers commonly found in the Japanese population. As a rule, pathological confirmation was obtained for the diagnosis of malignancy. When the recurrence of malignancy diagnosed before the onset of IgG 4-RD was observed during follow-up of IgG4-RD, it was not regarded as an occurrence of malignancy.

Age-stratified (according to 5-year age groups) and sexspecific data for the incidence of cancer in Japan (Center for Cancer Control and Information Services, National Cancer Center, Japan, period 2007) were used to determine the expected number of cases of malignancy in the cohort (12). The standardized incidence rate (SIR) was calculated as the ratio of the observed to the expected number of patients who developed malignancies. The $95 \%$ confidence interval (CI) of the SIR was estimated assuming a Poisson distribution (13).

Risk factors for malignancy were analyzed by comparing patients with and without malignancy. Possible risk factors included the age at onset of IgG4-RD, sex, number of lesions, diabetes mellitus, smoking and levels of IgG, IgG4 and $\mathrm{IgE}$ at the initial assessment.

Differences were analyzed using Student's $t$-test or Welch's $t$-test for continuous data and the $\chi^{2}$ test or Fisher's exact test as required for categorical data. Continuous variables are reported as the mean \pm SD. The cumulative incidence of malignancy was calculated according to the Kaplan-Meier method, and the effects of various variables on the risk of malignancy were evaluated using the log-rank test. Parameters proven to be significant in the univariate analysis were tested using a multivariate Cox proportional hazards model. All statistical analyses were performed using the JMP 7.0.1 software program (SAS Institute). All statistical tests were two-sided, and a $\mathrm{p}$ value of $<0.05$ was considered to be statistically significant.

This study was approved by the Institutional Review Board of our institution, and informed consent for all invasive procedures was obtained from each patient.

\section{Results}

\section{Patient characteristics}

In total, 113 patients with IgG4-RD (90 men and 23 women, mean age at onset: 65 years) were prospectively followed for $>6$ months. The mean and median follow-up periods were 73 and 64 months, respectively (range: 7-204 months). At the onset of IgG4-RD, pancreatic lesions (AIP), salivary and/or lacrimal gland lesions, periaortic lesions (including retroperitoneal lesions), extrapancreatic biliary lesions, pulmonary lesions, ophthalmologic lesions and pachymeninx lesions were observed in $82,22,20$, nine, three, two and one patient, respectively. Five patients had three IgG4related lesions, 16 patients had two lesions and 92 patients had one lesion. When the pancreatic lesions that appeared during follow-up were included, 95 patients (84\%) had AIP. All of these patients had type 1 AIP according to the ICDC. Twenty patients were followed without steroid treatment (ST). Twenty-seven patients were initially followed without ST for at least six months but received ST thereafter. In the remaining 66 patients, the disease was diagnosed and treated with steroids within six months after the onset. In principle, maintenance therapy with $2.5-7.5 \mathrm{mg} /$ day of prednisolone was administered for at least three years. Diabetes mellitus (DM), diagnosed before or around the diagnosis of IgG4$\mathrm{RD}$, was observed in 69 patients $(61 \%)$. A diagnosis of DM was made when the fasting blood glucose level was $\geq 126$ $\mathrm{mg} / \mathrm{dL}$ and/or the hemoglobin A1c (HbA1c) level was $\geq$ $6.5 \%$ (14). The extent of smoking was assessed based on 
Table 1. Clinical Profile of 14 Patients Associated with Malignancy

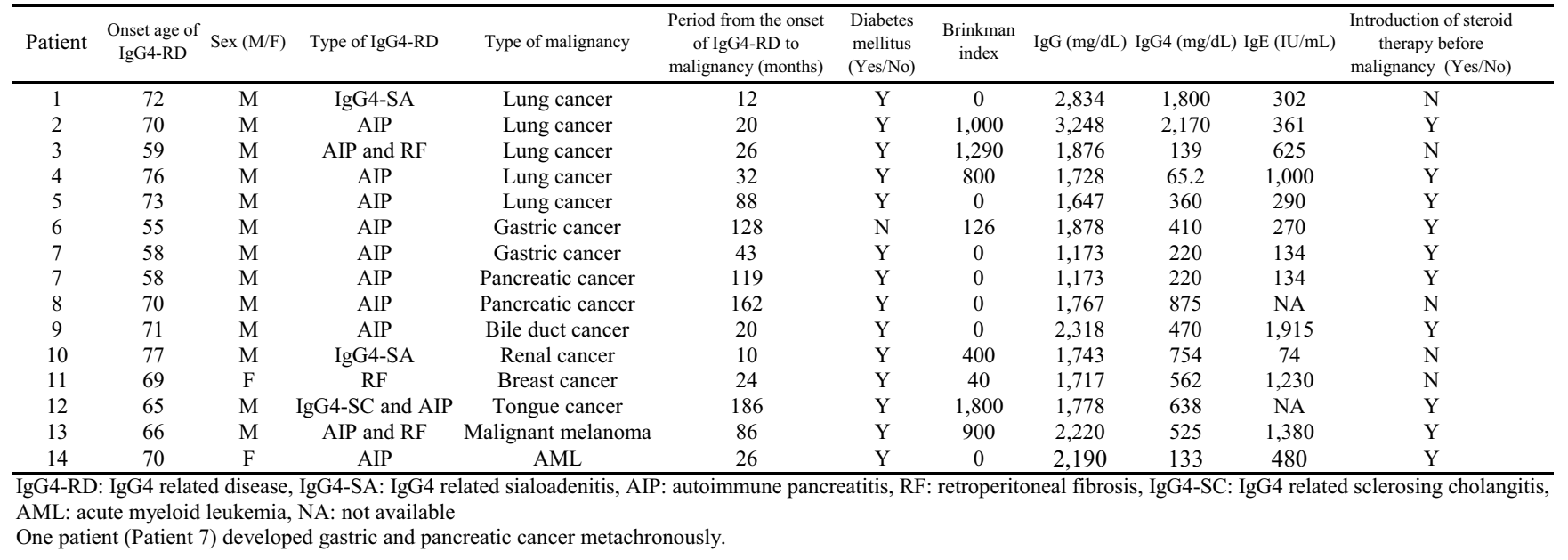

Table 2. Comparison between Patients with and without Malignancy

\begin{tabular}{cccc}
\hline Parameters & Malignancy $(+)(\mathrm{n}=14)$ & Malignancy $(-)(\mathrm{n}=99)$ & $\mathrm{p}$ value \\
\hline Onset Age (mean) of IgG4-RD & $67.9 \pm 6.6$ & $64.1 \pm 10.1$ & 0.179 \\
Onset Age $(\geq 65 \mathrm{y} /<65 \mathrm{y})$ & $11 / 3$ & $52 / 47$ & 0.087 \\
Sex (male/female) & $12 / 2$ & $78 / 21$ & 0.731 \\
Number of lesions $(1 />1)$ & $11 / 3$ & $81 / 18$ & 0.722 \\
Brinkmann index $($ mean) & $508 \pm 600$ & $487 \pm 607(\mathrm{n}=95)$ & 0.908 \\
Brinkmann index $(\geq 400 /<400)$ & $7 / 7$ & $45 / 50$ & 1.0 \\
DM (+/-) & $13 / 1$ & $56 / 43$ & 0.0084 \\
IgG (mg/dL) (mean) & $1,976 \pm 549$ & $1,999 \pm 775$ & 0.912 \\
IgG4 (mg/dL) (mean) & $645 \pm 620$ & $566 \pm 517$ & 0.604 \\
IgE (IU/mL) (mean) & $689 \pm 569(\mathrm{n}=12)$ & $729 \pm 1,161(\mathrm{n}=87)$ & 0.908 \\
\hline IgG4-RD: IgG4 related disease, DM: diabetes mellitus, Ig: immunoglobulin
\end{tabular}

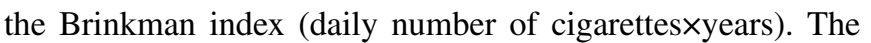
index was $<400$ in 61 patients $(54 \%)$.

\section{Development of malignancy during follow-up}

Fifteen malignancies developed in 14 patients (12\%) during follow-up (Table 1). Lung cancer developed in five patients, pancreatic cancer developed in two patients, gastric cancer developed in two patients and bile duct cancer, renal cancer, breast cancer, tongue cancer, malignant melanoma and acute myeloid leukemia developed in one patient each. One patient metachronously developed gastric cancer and pancreatic cancer 43 months and 119 months after the onset of IgG4-RD, respectively. To calculate the SIR of the total malignancies, the earlier episode of gastric cancer was counted in this patient. The mean and median periods between the onset of IgG4-RD and the initial development of malignancy were 62 and 29 months, respectively.

The SIR of the total malignancies was 1.04 (95\% CI 0.57-1.75). We also calculated the SIR of specific cancers that were observed in more than one patient. The SIR of lung cancer, pancreatic cancer and gastric cancer was 2.17 (95\% CI 0.70-4.99), 3.46 (95\% CI 0.40-11.9) and 0.75 (95\% CI 0.086-2.59), respectively. When the patients were limited to those with AIP $(n=95)$, the SIR of pancreatic cancer was 3.65 (95\%CI 0.42-12.5).

\section{Risk factors for malignancy in IgG4-RD patients}

The results of the comparison between the patients with and without malignancy are summarized in Table 2. The number of patients with DM was significantly greater in the malignancy $(+)$ group $(\mathrm{p}=0.0084)$. In addition, the number of patients with disease onset at an age of $\geq 65$ years tended to be greater in the malignancy $(+)$ group $(\mathrm{p}=0.087)$. According to the univariate analyses using the Kaplan-Meier method and log-rank test, "age at onset $\geq 65$ years" ( $\mathrm{p}=$ $0.029)$ and "association with DM" $(\mathrm{p}=0.031)$ were significant risk factors for the incidence of malignancy (Figure). A multivariate analysis using the Cox proportional hazards model was performed using these two parameters. Consequently, "age at onset $\geq 65$ years" was found to be the only risk factor (HR 3.82, 95\% CI 1.028-14.20) (Table 3).

It was difficult to evaluate the effects of ST on the incidence of malignancy because 27 patients had a follow-up period both with and without ST. In these patients, we focused only on the follow-up period prior to the introduction of ST. Forty-seven patients (35 men and 12 women, mean age at onset: 64 years) had a follow-up period without ST. The mean and median follow-up periods were 62 and 37 months, respectively (range: 7-201 months). Malignancy occurred in five patients (lung cancer in two patients, pancreatic cancer in one patient, renal cancer in one patient and 

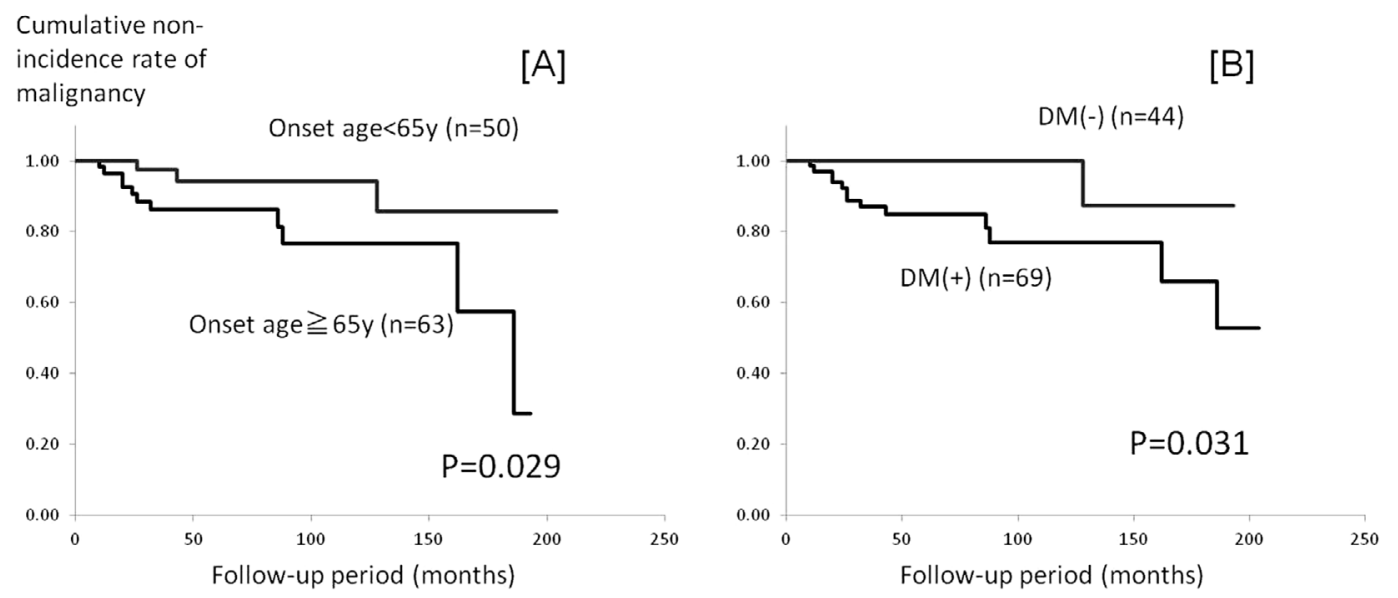

Figure. A) Kaplan-Meier curves comparing the non-incidence rate of malignancy between the "age of onset $<65$ years group" and "age of onset $\geq 65$ years group." B) Kaplan-Meier curves comparing the non-incidence rate of malignancy between the "DM (+) group" and "DM (-) group."

Table 3. Risk Factors for Malignancy in IgG4RD (Multivariate Analysis by the Cox Proportinal Hazards Model)

\begin{tabular}{lcc}
\hline Parameters & Hazard ratio $(95 \% \mathrm{CI})$ & $\mathrm{p}$ value \\
\hline Onset age $(\geq 65 \mathrm{y})$ & $3.82(1.03-14.2)$ & 0.045 \\
Diabetes mellitus & $6.71(0.87-51.5)$ & 0.067 \\
\hline
\end{tabular}

breast cancer in one patient). The SIR of the total malignancies in this cohort was 1.05 (95\% CI 0.34-2.40), which did not differ from the SIR of the total cohort (1.04). Therefore, ST does not appear to be a significant risk or protective factor for the incidence of malignancy in IgG4-RD patients.

\section{Discussion}

This is the largest-scale report of a systematic estimation of the incidence of malignancy in patients with IgG4-RD using a long-term follow-up period. In light of the chronic inflammation and frequent DM observed in such patients, it is anticipated that there would be a higher incidence of malignancy in patients with IgG4-RD. However, the incidence of total malignancies was similar to that observed in the age- and sex-matched Japanese controls. Evaluating specific malignancies is difficult because the number of cases is small. The SIR of pancreatic cancer (3.40) appeared to be a little high; therefore, it should be evaluated in a larger scale study.

Since we aimed to clarify whether IgG4-RD can cause malignancy, we selected patients with IgG4-RD who developed malignancy during the follow-up period. Therefore, we excluded seven patients in whom malignancy was diagnosed $\leq 6$ months before or after the onset of IgG4-RD (gastric cancer in two patients, lung cancer in one patient, renal cancer in one patient, esophageal cancer in one patient, prostate cancer in one patient and concomitant pancreatic and colon cancer in one patient) and six patients whose follow-up pe- riod was $\leq 6$ months. Including these 13 patients, the incidence of total malignancies is $17 \%(21 / 126)$, which seems rather high. Considering the relationship between IgG4-RD and malignancy, it is very difficult to evaluate patients who develop IgG4-RD and malignancy around the same time (15). Of these seven patients, IgG4-RD was diagnosed during the close examination of malignancy in five cases. Because our institute is a tertiary hospital where many cancer patients are treated, various diseases may be diagnosed during the close examination of malignancy. In other words, a hospital examining a higher number of cancer patients may have a higher number of patients with IgG4-RD and malignancy. Although "seven patients" seems to be a comparatively large number, the effect of such selection bias cannot be denied.

As for the exclusion period of "six months," we must admit that it was arbitrarily defined and may be insufficient. Unfortunately, this problem appears to be unavoidable. Selecting one or two years is possible instead of six months; however, whichever period is chosen will be arbitrary. If a longer period is adopted, the calculated SIR for total malignancies becomes smaller because more patients with malignancies are excluded. For example, if one year is adopted, the SIR for total malignancies is 0.90 (95\%CI 0.47-1.56). Therefore, we chose to adopt a short period (six months) and obtained a conservative calculation of the SIR for our position.

Moreover, in this study, the follow-up period for calculating the SIR of total malignancies in the patients who developed malignancy was defined as the period between the onset of IgG4-RD and the occurrence of malignancy. Because calculating the SIR becomes impossible if patients whose follow-up period is zero are included, it is difficult to add patients who developed IgG4-RD and malignancy concurrently in the analysis.

Two recent studies evaluating the SIR support the association between $\operatorname{IgG} 4-\mathrm{RD}$ and total malignancies $(16,17)$. Yamamoto et al. reported the SIR for total malignancies to 
be 3.8 in their series, which consisted primarily of patients with Mikulicz's disease (16), while Shiokawa et al. reported the SIR to be 2.7 in their series, which consisted of AIP patients only (17). Both studies concluded that the SIR was significantly high. However, both series included cases in which malignancies and IgG4-RD were diagnosed concurrently. In these two studies, the follow-up period of the patients who developed malignancy was not defined as the period between the onset of IgG4-RD and the occurrence of malignancy, but rather as the simple follow-up period, namely, the period between the diagnosis of IgG4-RD and the day of the last visit or death. If the SIR is calculated using this method, it follows that the timing of the occurrence of malignancy does not matter, whether it is at the time of diagnosis of IgG4-RD or during follow-up. This calculating method may overestimate the SIR when there are many patients in whom malignancy and IgG4-RD are diagnosed concurrently and the follow-up period is short. Therefore, in order to examine whether IgG4-RD causes malignancy, we believe that such cases should be excluded from the analysis. If IgG4-RD really causes malignancy, the results should be the same, regardless of the inclusion or exclusion of such cases.

In Shiokawa's series, 18 cancers were found in 108 AIP patients; however, eight cancers were diagnosed concurrently (17). Regarding this point, the authors speculated that AIP may develop as a paraneoplastic syndrome in some patients. They added that immunoreactions to cancer may induce AIP. This hypothesis is interesting and plausible; however, there appears to be inadequate evidence to support it at present. The problem of selection bias also cannot be denied. We aimed to clarify whether IgG4-RD is a risk factor for malignancy (= could cause malignancy) and cannot confirm or deny the authenticity of this hypothesis. For reference, we calculated the SIR of total malignancies using the same method as Shiokawa et al. That is, the data of all 126 patients were used for the analysis. As a result, the SIR of total malignancies was 1.56 (95\%CI 0.99-2.34), which was barely significant. Our data and Shiokawa's data may be similar; however, the conclusions are quite opposite because we focused only on whether IgG4-RD can cause malignancy, while they considered both the possibility that "IgG4RD causes malignancy" and "malignancy causes IgG4-RD."

A univariate analysis of the risk factors for malignancy in patients with IgG4-RD revealed that "age at onset of IgG4$\mathrm{RD} \geq 65$ years" and "association with DM" were significant risk factors, with age at onset remaining significant in the multivariate analysis. These results are not unexpected $(12,18)$. Assuming that IgG4-RD itself is related to the increased incidence of malignancy, it is expected that IgG and IgG4, which reflect the disease activity (19), would be risk factors for malignancy. Treatment without steroids may increase the risk of malignancy. Because such results were not obtained, the hypothesis is not true. We also expected that $\operatorname{IgE}$ would be a protective factor for malignancy given the reports of an inverse association between the level of $\operatorname{IgE}$ and cancer (20-22). However, this assumption also does not appear to be true.

The limitation of this study is that specifying the onset time of IgG4-RD is sometimes difficult. If there were preceding relevant symptoms before the final diagnosis of IgG4-RD, the timing of the appearance of the symptoms was regarded as the onset time. However, in patients without any symptoms, the onset time was regarded as the time of diagnosis. As for malignancies, the onset time was substituted for the time of diagnosis. Strictly speaking, it is difficult to determine the exact time of onset of IgG4-RD or malignancy in any case.

In conclusion, it is reasonable to conclude that IgG4-RD is not associated with an increased incidence of total malignancies. A larger scale study is needed to evaluate the incidence of specific cancers.

\section{The authors state that they have no Conflict of Interest (COI).}

\section{Acknowledgement}

This work was partially supported by the Research Program on Intractable Disease (Research on IgG4-related Disease) provided by the Ministry of Health, Labour and Welfare of Japan.

\section{References}

1. Sah RP, Chari ST. Long term prognosis in IgG4-related systemic disease. Curr Immunol Rev 7: 239-245, 2011.

2. Hirano $\mathrm{K}$, Tada $\mathrm{M}$, Isayama $\mathrm{H}$, et al. Long-term prognosis of autoimmune pancreatitis with and without corticosteroid treatment. Gut 56: 1719-1724, 2007.

3. Kubota K, Iida H, Fujisawa T, et al. Clinical factors predictive of spontaneous remission or relapse in cases of autoimmune pancreatitis. Gastrointest Endosc 66: 1142-1151, 2007.

4. Takuma K, Kamisawa T, Tabata T, Inaba Y, Egawa N, Igarashi Y. Short-term and long-term outcomes of autoimmune pancreatitis. Eur J Gastroenterol Hepatol 23: 146-152, 2011.

5. Yoshida K, Toki F, Takeuchi T, Watanabe S, Shiratori K, Hayashi $\mathrm{N}$. Chronic pancreatitis caused by an autoimmune abnormality. Proposal of the concept of autoimmune pancreatitis. Dig Dis Sci 40: 1561-1568, 1995.

6. Members of the Criteria Committee for Autoimmune Pancreatitis of the Japan Pancreas Society. Diagnostic criteria for autoimmune pancreatitis by the Japan Pancreas Society. Suizo (J Jpn Panc Soc) 17: 585-587, 2002 (in Japanese).

7. Okazaki K, Kawa S, Kamisawa T, et al. Clinical diagnostic criteria of autoimmune pancreatitis: revised proposal. J Gastroenterol 41: 626-631, 2006.

8. Otsuki M, Chung JB, Okazaki K, et al. Asian diagnostic criteria for autoimmune pancreatitis: consensus of the Japan-Korea Symposium on Autoimmune Pancreatitis. J Gastroenterol 43: 403-408, 2008.

9. Chari ST, Takahashi N, Levy MJ, et al. Diagnostic strategy to distinguish autoimmune pancreatitis from pancreatic cancer. Clin Gastroenterol Hepatol 7: 1097-1103, 2009.

10. Shimosegawa T, Chari ST, Frulloni L, et al. International consensus diagnostic criteria for autoimmune pancreatitis: guidelines of the International Association of Pancreatology. Pancreas 40: 352358, 2011.

11. Umehara H, Okazaki K, Masaki Y, et al. Comprehensive diagnostic criteria for IgG4-related disease (IgG4-RD), 2011. Mod Rheumatol 22: 21-30, 2012. 
12. Matsuda $T$, Marugame $T$, Kamo $K$, et al. Cancer incidence and incidence rates in Japan in 2006: based on data from 15 populationbased cancer registries in the monitoring of cancer incidence in Japan (MCIJ) project. Jpn J Clin Oncol 42: 139-147, 2012.

13. Ury HK, Wiggins AD. Another shortcut method for calculating the confidence interval of a Poisson variable (or of a standardized mortality ratio). Am J Epidemiol 122: 197-198, 1985.

14. The Committee of Japan Diabetes Society on the diagnostic criteria of diabetes mellitus. Report of the committee on the classification and diagnostic criteria of diabetes mellitus. J Jpn Diabetes Soc 53: 450-467, 2010 (in Japanese).

15. Kawakubo $\mathrm{K}$, Tada $\mathrm{M}$, Isayama $\mathrm{H}$, et al. Incidence of extrapancreatic malignancies in patients with intraductal papillary mucinous neoplasms of the pancreas. Gut 60: 1249-1253, 2011.

16. Yamamoto M, Takahashi H, Tabeya $T$, et al. Risk of malignancies in IgG4-related disease. Mod Rheumatol 22: 414-418, 2012.

17. Shiokawa M, Kodama Y, Yoshimura K, et al. Risk of cancer in patients with autoimmune pancreatitis. Am J Gastroenterol 108: 610-
617, 2013.

18. Hirakawa Y, Ninomiya T, Mukai N, et al. Association between glucose tolerance level and cancer death in a general Japanese population: the Hisayama Study. Am J Epidemiol 176: 856-864, 2012.

19. Hirano K, Tada M, Isayama H, Sasahira N, Koike K. Significance of measuring $\mathrm{IgG}$ and $\mathrm{IgG} 4$ during follow-up of autoimmune pancreatitis. Pancreas 40: 788-791, 2011.

20. Schwartzbaum J, Ding B, Johannesen TB, et al. Association between prediagnostic IgE levels and risk of glioma. J Natl Cancer Inst 104: 1251-1259, 2012.

21. Hirano K, Tada M, Mizuno S, et al. Lower incidence of biliary carcinoma in patients with primary sclerosing cholangitis and high serum levels of Immunoglobulin E. Clin Gastroenterol Hepatol 10: 79-83, 2012.

22. Van Hemelrijck M, Garmo H, Binda E, et al. Immunoglobulin E and cancer: a meta-analysis and a large Swedish cohort study. Cancer Causes Control 21: 1657-1667, 2010.

(C) 2014 The Japanese Society of Internal Medicine http://www.naika.or.jp/imonline/index.html 